\title{
A Performance Measurement System to Manage CEN Operations, Evolution and Innovation
}

\author{
Raul Rodriguez-Rodriguez, Juan-Jose Alfaro-Saiz, and Maria-Jose Verdecho \\ Universitat Politècnica de València, Camino de Vera s/n, 46022, Valencia, Spain \\ \{raurodro, jalfaro, mverdecho\} @cigip.upv.es
}

\begin{abstract}
Nowadays, many enterprises collaborate forming a collaborative enterprise network in order to achieve competitive and sustainable advantages. These collaborative enterprise networks are operating many times under a glocal approach, which means that they have less time to react, to properly evolve and more challenges to face, mainly the fact of operate both locally (product design, sales) and globally (manufacturing, distribution). In this context, collaborative enterprise networks need to manage not only their classic operations (costs, quality, response, flexibility, etc) but they also need to integrally incorporate to their management and decision-making systems their collaborative practices regarding evolution and innovation. Then, this work presents an evolved performance measurement system to manage collaborative enterprise networks operations including co-innovation and co-evolution practices. Such a system has got four phases and, through the identification and quantification of relationships among performance elements, it offers a new management approach, which will provide collaborative enterprise network decision-makers with additional and meaningful information.
\end{abstract}

Keywords: Collaborative enterprise network, operations, glocal, co-innovation, co-evolution.

\section{Introduction}

Currently, it is a common practice to do business in globalized markets under a collaborative approach, constituting a Collaborative Enterprises Network (CEN). Additionally, global markets dictate conditions difficult to achieve at the same time such as highly customized products/services (even locally customized), to be offered in high amounts (products/services produced and supplied from geographically distributed enterprises), within very short lifecycles and volatile business environments. This approach represents what it is widely known as Glocal approach: to think globally and to act locally. From a CEN point of view, this implies to coordinate many complex operations at a global level in order to supply products and services that are responding to the gathered locally identified customers' needs and tastes. The associated challenges are, even nowadays with all the technological and managerial advances at disposal, of enormous proportions. Further, if taking into consideration the timely factor, it seems evident that the customers' needs and tastes, 
the market evolution and trends and the own CEN are dynamic and should evolve over time. Focusing on the CEN level, its individual partners should be able to evolve together in order to be able to adapt to both customer and markets changes for not getting dismissed from the marketplace, what it can be seen as a co-evolution process of the CEN. In addition to this co-evolution issue, if a CEN wants to be competitive over time, it must be able to design and offer innovative products and services, which is known as co-innovative products and services process. In this process, customers could also be invited to participate during the innovation process, adopting new roles and even being formally part of the CEN [1]. But a CEN cannot be only a source of locally customized products and services to be produced and supplied in a global basis but it must also make it efficiently. This implies to control and manage the CEN's operations in order that the CEN will be cost-efficient, robust, with a high degree of responsiveness, etc. Then, tools and mechanisms that will help to properly manage CEN operations from a dynamic point of view are needed. This paper deals with the co-evolution issue from a decision-making point of view, presenting a methodology that will be able to improve both CEN's short-term decisions and CEN's strategic long-term definition.

The next section looks at the academic literature that have dealt, in some manner, with this approach, setting the basis for the framework developed in this work: A performance measurement system to manage CEN operations, whose base is to identify and quantify the existing relationships among performance elements, it is presented in the point 3 . Lastly, point 4 highlights some findings from a real application of the proposed system.

\section{Background}

\subsection{CEN Co-Evolution}

As stating above, a CEN is dynamic and it changes over time, not only from an external point of view (due to exogenous factors) but also from an internal one (due to endogenous factors). All these factors are influencing over the CEN's lifecycle whose main states are next explained [2]. After a first phase of Creation where the partners are stated together with their duties, responsibilities, etc., the Operations phase takes place. It is within this phase when the CEN develops its main tasks of product/service design, manufacturing, distributing, etc. This is the natural state of a CEN. Within this Operations phase, it is the sub-phase called Evolution that takes place when there are non-significant changes in members, roles, etc. [2]. Nowadays, CEN evolution is not seen in isolation but it must be carried out under a collaborative approach, forming the called co-evolution. The ability to get adapted to environmental and internal changes within shorter and shorter product/services lifecycles, together with the need to efficiently and timely respond to changes makes individual evolutions of CEN members not longer efficient and demands a collaborative response. Therefore, there is a crescent need to manage such a co-evolution of a CEN from an integrated point of view, assessing its degree and its impact over the whole CEN's operations. Finally, a 
CEN may experiment a Metamorphosis when a great change in the CEN takes place or a Dissolution that it is when the CEN does not exit anymore [2].

Then, co-evolution practices and actions are becoming more and more important and, then, they should be incorporated in an integrated manner to the CEN's decisionmaking tools and mechanisms. It is important to point out that this work deals with all types of CEN, as long as they have previously, as starting point, formally defined a set of strategic objectives and associated KPIs.

\subsection{CEN Co-Innovation}

One of the most recent collaborative forms is the collaborative innovation or coinnovation. Within this co-innovation form, [1] mentions the next relevant features/elements:

- Understanding the innovation process.

- Brainstorming.

- Value identification.

- Mechanisms for safety/protection.

- Trust management.

- Supporting sharing activities.

All of these are important and to take into account when undertaking collaborative innovation practices. From an assessment point of view, the value identification is the one that has more to do with performance measurement systems. Further, at the CEN context, the value identification is carried out in a subjective way, as it is presented in [3]. They develop a set of qualitative assessments methods for assessing the called Value Systems in a CEN by applying fuzzy casual maps. The objective is to integrate multiple aspects regarding the alignment of value systems within a CEN, where different member has got different values and preferences.

Additionally, when looking deeper at the glocal concept, it is evident that nowadays the customer demands products/services that are more customized than ever. If these products/services are going to be operated through a CEN, this implies that new efforts regarding, among others, co-innovation should be done. Then, [1] identifies the called "customer involvement" as one of the most important task to accomplish. This implies that not only the CEN members, but also the customers, should participate when innovation is at stake. It is even mentioned that the customer could become a member of the CEN, extending therefore the approach of customers participating on product design of individual companies.

Therefore, co-innovation practices are an important part of the current CEN's operations and, therefore, they need to be integrally taken into account within the CEN's management system.

\subsection{CEN Operations}

Regarding a CEN's operations: how can be these controlled and managed in the current business ecosystem? There are numerous approaches developed during the 
last years that deal with managing a CEN's performance that is already working under operation $[4,5,6,7]$. All these frameworks manage performance by linking the stages of both creation and operation through the whole CEN lifecycle. However, none of them provides any direction on how to measure and manage overtime a CEN's operations by taking into account the results achieved by the performance measurement in the previous period as an input for the current period definition. Additionally, these works do not establish any meaningful link between the defined performance elements (mainly between the defined strategic objectives and the performance indicators), when it is widely accepted that these elements are intrinsic linked [8]; further, there is not yet any framework at the CEN context that identifies and quantifies these relationships. Additionally, a CEN's operations should be not taken as isolated in the current glocal business environment. Derived from the previously introduced, co-evolution and co-innovation are key issues that are directly and strongly impacting over a CEN's operations performance. Therefore, this work has gathered all this together and, in the next point, presents an innovative and evolved performance measurement system to manage CEN operations, integrating not only the classic operations such as cost, quality, responsiveness, etc, but also performance elements regarding the CEN's degree of co-evolution and co-innovation.

\section{A Performance Measurement System to Manage CEN Operations (PMS-CEN OP)}

This point presents the developed Performance Measurement System to manage CEN operations (PMS-CEN OP). The main tasks to be developed within each of the phases are next introduced:

- Phase 1. Definition of the CEN's targets and associated responsibilities. In this first phase, the accorded CEN targets are defined. This is a complex process, as all the different CEN partners must reach an agreement at both the strategic and the associated operational levels. Then, they must agree on the strategic objectives (called in this paper indistinctly targets) to be pursued during the next time-period (usually one year). It should be kept in mind that a given individual member of the CEN might have some own individual strategic objectives that are in conflict with some of the strategic objectives that the CEN aims to define. Negotiation processes must then be carried out, as this initial phase is key for the CEN's success. Regarding the CEN's strategic objectives, these will be defined according to the CEN's strategic line that needs to be covered in this precise moment. For instance, if the CEN wants to compete under a strategic line of "operational excellence", the strategic objectives to be defined will have to be about reducing the CEN's operational time, augmenting the CEN's responsiveness, augmenting the CEN's robustness, etc. Additionally, the PMS-CEN OP allows CEN decision-makers to define strategic objectives that have to do with both co-innovation and with $\mathrm{co}$ evolution. These objectives can be defined under a more general context, 
and should focus on issues such as the CEN's co-innovative practices, success, members participating, degree of response, etc., and also on the CEN's co-evolution state, tasks, adaptations, etc. This will provide a complete definition of strategic objectives, moving then to define associate key performance indicators (KPIs). These KPIs will check out whether the associated strategic objectives have been reached or not. The KPIs will be of different nature regarding their frequency and units of measurement. The final task within this phase is to allocate responsibilities of each CEN member over each of the defined objectives. This is an innovative and important point in order to properly carry out the last phase of this framework. If the responsibilities of each member is well limited and defined and everybody has agreed on this point, when the leading (or cause) performance elements have been identified and quantified within the PMS-CEN OP, the actions to be taken will be more easily defined and assigned.

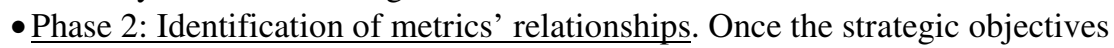
and associated KPIs have been defined, the PMS-CEN OP starts to operate and the KPIs start to measure and store data. This data contains important information, which is usually ignored, as it reveals patterns and behaviors that, when extended to the KPIs and strategic objectives levels, it is the source of valuable additional information. Then, it is possible to think in identifying relationships, in a quantitative manner, between the defined KPIs. Different techniques may be used depending of the number of observations, number of KPIs, missing values, etc. Some techniques that might be used are the well-known Multi-Criteria Decision Aid techniques; being widely used the Analytic Network Process [9]. On the other hand, it is also possible to apply statistical techniques such as correlation analysis, multiple regression or principal component analysis. The results are sets of cause-results KPIs. This mean that, when a cause KPI changes in value over time it makes that a result KPI changes too. From a decisional point of view, this means that this framework offers the possibility of focusing on less KPIs (the cause ones) when making decisions. Further, it allows bringing together relationships between all the defined KPIs, operational, co-innovation and co-evolution ones.

- Phase 3: Projection of relationships to targets/strategic objectives level. Additionally, the found relationships can also be projected towards the associated strategic objectives, carrying then out similar reasoning but at the strategic level (Figure 1 illustrates this tasks). This implies that, with the application of this framework, it might turn out that, for example, a certain co-evolution KPI (associated to the strategic objective of "Degree of adaptability to business requirements of the CEN") is the cause of a KPI that measure the global CEN cost (and associated to a strategic objective of "Reducing the CEN cost in a 10\%"), when projecting these cause-effect relationships to the targets level, it will be possible to conclude that positive changes on the CEN's degree of adaptability are 
leading to reach a lower CEN cost. This type of analysis is an innovative one at the CEN context and it provides decision-makers with important and additional information.

- Phase 4: Allocation of responsibilities. It is necessary to keep in mind that the PMS-CEN OP works at the CEN level but that there another lower level: the individual CEN members level. Then, once that the cause-effect relationships between performance elements have been establish and decisions made, this phase offers an innovative approach of responsibility allocation between the different members of the CEN.

Finally, it is important to point out that all the efforts carried out when applying the PMS-CEN OP should be taken into account in the next period when defining again the CEN's performance elements.

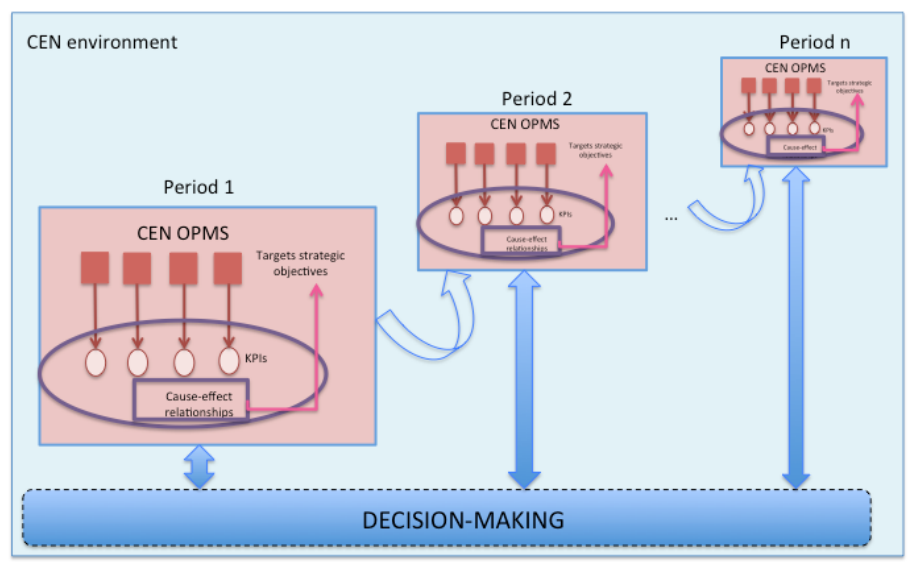

Fig. 1. PMS-CEN OP

\section{Application}

Generally speaking, it is possible to enumerate a list, from a general viewpoint, of useful KPIs to apply to measure CEN operations. Therefore, these KPIs are susceptible of being used at this context. Some examples of KPIs are the following: "Number of collaborative methodologies for improving resource efficiency and overall added value", "Definition of best practices and guidelines to re-engineer intercompany operations collaboratively in order reduce and eliminate wastes", "Number of business opportunities identified within the life-cycle of manufacturing products", "Number of supply chain reconfigurations", "Number of business model innovations", "Expected improvement of the overall equipment effectiveness/functional unit in the value chain", etc. However, each CEN will have different needs in terms of operations and defined performance elements as well as different maturity degree regarding the necessary expertise to implement the PMSCEN OP. 
This point aims to be illustrative in terms of presenting the main results coming from applying the PMS-CEN OP to a CEN that manufactures and supplies bathroom furniture. Then, as a result of applying phases 1,2 and 3 several important (with strong intensity) targets cause-effect relationships were found. Further, 17 important cause-effect relationships were found and studied, being the different effect KPIs from the Financial (7), Customer (2), Internal Processes (6) and Learning and Growth (2) perspectives. Proper decision-making analyses were carried out for each of these 17 cause-effect relationships in order to improve the additional information to make better decisions at the CEN level and, extensively, being more efficient.

For instance, it was identified that the strategic objective F1 (Increment 5\% net profit) was directly affected by F3 (decrement in a 10\% product development costs), C5 (Increment in a 15\% the percentage of new products based on customer input/design) and P1 (Decrement stock variability in a 15\%). This means that the CEN should focus on achieving F3, C5 and P1 in order to successfully achieve F1. As a result of allocating responsibilities, it was clearly stated which CEN members should focus more on achieving these cause strategic objectives, resulting on a more coordinated and smooth actions. Since F1 was the most important strategic objective, it was decided to include it again in the next period PMS definition and, therefore, F1's cause strategic objectives (F3, C5 and P1) should be also included in such next definition, providing dynamicity to such a definition process.

This approach can be applied to any CEN that has a fully operating PMS in terms of defined objective and associated performance indicators. More applications would also enrich this initial approach and would, therefore, help to overcome the drawbacks found during its applications, being the main one the lack of knowledge of people in the CEN to apply and interpret the additional information that this evolved PMS points out. Additionally, it would be of great importance to set up some mechanisms that would make smoother and easier both the negotiation and definition activities within the CEN members.

\section{Conclusions}

This work has presented an innovative evolved performance measurement system to manage CEN operations (PMS-CEN OP). It includes not only the classic operations but it also incorporates both the co-innovation and co-evolution practices within the PMS-CEN OP, as in current competitive markets and under the glocal perspective these practices must be integrally taken into account and introduced within the CEN's management systems. Additionally, the PMS-CEN OP is dynamic (the main results of applying it in a certain period should be an input to the next period's definition) and it applies quantitative techniques in order to identify and quantify cause-effect relationships among the performance elements.

In terms of future research lines, it can be mentioned the need of further implementing the PMS-CEN OP to other CENs: Product, service, service-product. This will allow enriching this first approach and, therefore, it will turn into a better management tool. 
Acknowledgements. This work has been developed within the research project called "Design of business scenarios to improve the efficiency and management of industrial supply chain" (Ref.: GV/2013/045) funded by the Generalitat Valenciana.

\section{References}

1. Camarinha-Matos, L.: Collaborative networked organizations: Status and trends in manufacturing. Annual Reviews in Control 33, 199-208 (2009)

2. Camarinha-Matos, L., Afsarmanesh, H., Galeano, N.: Collaborative Networked Organizations: Concepts and practice in manufacturing enterprises. Computers \& Industrial Engineering 57, 46-60 (2009)

3. Macedo, P., Camarinha-Matos, L.: A qualitative approach to assess the alignment of Value Systems in collaborative enterprises networks. Computers \& Industrial Engineering 64, 412-424 (2013)

4. Bititci, U.S., Mendibil, K., Martinez, V., Albores, P.: Measuring and managing performance in extended enterprises. International Journal of Operations \& Production Management 25(4), 333-353 (2005)

5. Folan, P., Browne, J.: Development of an Extended Enterprise Performance Measurement System. Production Planning and Control 16(6), 531-544 (2005)

6. Alfaro, J., Ortiz, A., Rodríguez, R.: Performance measurement system for Enterprise Networks. International Journal of Productivity and Performance Management 56(4), 305334 (2007)

7. Francisco, R.D., Azevedo, A.: Dynamic Performance Managemen. In: Business Networks Environment. In: Digital Enterprise Technology. Springer US (2007)

8. Rodriguez-Rodriguez, R., Alfaro-Saiz, J.J., Ortiz-Bas, A.: Quantitative relationships between key performance indicators for supporting decision-making processes. Computers in Industry 60, 104-113 (2009)

9. Saaty, T.L.: The analytic network process: decision-making with dependence and feedback. RWS Publications, Pittsburgh (1996) 